\title{
RECIPIENTES E SUBSTRATOS NA PRODUÇÃO DE MUDAS DE Maytenus ilicifolia E Apuleia leiocarpa ${ }^{1}$
}

\author{
POT AND SUBSTRATE ON SEEDLING PRODUCTION \\ OF Maytenus ilicifolia AND Apuleia leiocarpa ${ }^{1}$
}

\author{
Fernando Teixeira Nicoloso $^{2}$ Roni Paulo Fortunato ${ }^{3}$ Flavio Zanchetti ${ }^{4}$ \\ Luciano Fábio Cassol $^{4}$ Sonia Maria Eisinger ${ }^{5}$
}

\section{RESUMO}

A cancorosa (Maytenus ilicifolia Martius ex Reissek) e a grápia (Apuleia leiocarpa (Vogel) Macbride) encontram-se em processo de dizimação devido à exploração excessiva dos recursos naturais e, portanto, estudos relacionados aos métodos de propagação dessas espécies são relevantes. $O$ objetivo deste trabalho foi avaliar diferentes recipientes e o tipos de substrato de cultivo na produção de mudas de cancorosa $e$ grápia. Os tratamentos consistiram de uma combinação bifatorial (7x3), representados por sete tamanhos de recipientes: saco plástico (tamanho pequeno, médio e grande, respectivamente, 1515,3064 e $6031 \mathrm{~cm}^{3}$ ), laminado de madeira (tamanho pequeno, médio e grande, respectivamente, 497, 829 e $\left.1161 \mathrm{~cm}^{3}\right)$ e tubete (tamanho único, modelo cilíndrico, $50 \mathrm{~cm}^{3}$ ) e três tipos de substratos: solo (horizonte A de um Podzólico vermelho amarelo), solo + casca de arroz carbonizada e areia média + casca de arroz carbonizada, na proporção 1:1 v/v. A semeadura de ambas as espécies foi realizada no dia 12 de dezembro de 1997. Aos 135 dias do período experimental, sob condições de temperatura ambiente, foram avaliadas a percentagem de sobrevivência das mudas, altura da planta, diâmetro do caule, comprimento do sistema radicular, peso da matéria seca de raízes, do caule, das folhas e total da planta, bem como a caracterização física dos substratos. A combinação do recipiente saco plástico de tamanho pequeno e substrato solo + casca de arroz carbonizada apresenta características adequadas à produção de mudas de cancorosa. A combinação dos recipientes saco plástico de tamanho médio e grande e o laminado de madeira grande combinados com os substratos solo + casca de arroz carbonizada e solo isoladamente apresentam características adequadas à produção de mudas de grápia.
Palavras-chave: cancorosa, grápia, tamanho de recipiente, substrato, produção de mudas.

\section{SUMMARY}

The species 'cancorosa' (Maytenus ilicifolia Martius ex Reissek) and 'grápia' (Apuleia leiocarpa (Vogel) Macbride) have been considered two endangered Brazilian forest trees due to the excessive exploration of natural resources and, therefore, information about suitable methods for their propagation is relevant. The aim of this work was to evaluate different pots and the types of substrate in the production of seedlings of 'cancorosa' and 'grápia'. The treatments followed a bifactorial scheme (7x3), represented by seven pot sizes: plastic bag (small, medium, and large size, respectively, 1515, 3064, and $\left.6031 \mathrm{~cm}^{3}\right)$, laminated wooden pot (small, medium and large size, respectively, 497, 829, and $1161 \mathrm{~cm}^{3}$ ) and plastic tube (only one size, cylindrical model, $50 \mathrm{~cm}^{3}$ ), and three types of substrates: soil alone (profile A from a red yellow podzolic soil), soil + carbonized rice husk, and medium sand + carbonized rice husk, 1:1 v/v). The sowing of both species was effectuated on 12 December 1997 and the experiment lasted for 135 days under ambient temperature. At this date, the percentage of plant survival, plant height, stem diameter $0,5 \mathrm{~cm}$ aboveground, length of the root system, dry weight of roots, stem, leaves, and total of the plant, as well as the physical properties of the substrates were analyzed. The small plastic bag, in combination with soil + carbonized rice husk, shows a suitable condition on the seedlings production of 'cancorosa'. The medium and large plastic bags and the large wooden pot, in combination with soil + carbonized rice husk and soil alone, present an adequate condition on the seedlings production of 'grápia'.

Key words: cancorosa, grápia, pot size, substrate, seedling production.

\footnotetext{
${ }^{1}$ Projeto financiado pelo PIBIC/CNPq.

${ }^{2}$ Engenheiro Agrônomo, PhD., Professor Adjunto do Departamento de Biologia, Centro de Ciência Naturais e Exatas (CCNE), Universidade Federal de Santa Maria (UFSM), 97105-900, Santa Maria, RS. E-mail: nicoloso@ sm.conex.com.br Autor para correspondência.

3 Acadêmico do curso de Agronomia, UFSM, Bolsista PIBIC/CNPq.

${ }^{4}$ Acadêmico do curso de Engenharia Florestal, UFSM, Bolsista PIBIC/CNPq.

${ }^{5}$ Biólogo, Doutor, Professor Adjunto do Departamento de Biologia, CCNE, UFSM.
} 


\section{INTRODUÇÃO}

A cancorosa ou espinheira-santa (Maytenus ilicifolia Martius ex Reissek) é pertencente à família Celastraceae e conhecida na medicina popular. É uma planta perene, arbustiva, nativa do sul do Brasil, norte da Argentina, Uruguai e Paraguai, ocorrendo em orlas de bosques e capões, matas ciliares, em nascentes de arroios. Exige solos férteis com bom teor de matéria orgânica e adapta-se a excessos de umidade temporários (CASTRO \& CHEMALE, 1995). GEOCZE et al. (1988) observaram, em um grupo de pacientes com úlcera gástrica, que o liofilizado da cancorosa reduziu a sintomatologia da doença sem apresentar efeitos colaterais. Resultados semelhantes foram obtidos por CARLINI \& BRAZ (1988) em população de ratos, onde Maytenus ilicifolia e M. aquifolium exerceram efeito antiúlceroso, reduzindo o número de úlceras a menos de $20 \%$ dos valores da testemunha.

A grápia (Apuleia leiocarpa (Vogel) Macbride) é uma planta leguminosa que ocorre desde alguns estados do Nordeste brasileiro até o Rio Grande do Sul, estendendo-se ao leste do Paraguai e à Província de Missiones, na Argentina. Segundo BRASIL (1973), no estado do Rio Grande do Sul, a área ocupada por essa espécie é pequena, encontrando-se nas partes mais altas do Nordeste do planalto, onde o solo é fértil, profundo e úmido e, em pontos esparsos, na Depressão Central. A grápia apresenta grande porte, servindo como excelente fonte de matéria-prima para serrarias. Para MATTOS \& GUARANHA (1983), a madeira da grápia apresenta ótima qualidade, sendo usada para marcenaria, esquadrias, postes para cercas e para outros fins.

Devido ao aumento na exploração dessas espécies, verifica-se um crescente esgotamento das reservas naturais. Apesar disso, pouco interesse tem sido demonstrado quanto à pesquisa de métodos visando à produção de mudas.

O uso de recipientes na produção de mudas de inúmeras espécies vegetais vem sendo empregado na maioria dos viveiros e, dentre as suas vantagens, salientam-se: (i) controle eficaz de fungos e nematóides; (ii) possibilidade de acelerar o processo de produção de mudas através do uso de substratos específicos; (iii) bom controle da condição nutricional; (iv) obtenção de mudas com sistema radicular bem desenvolvido, sem traumatismos e lesões, com facilidade no transplante; (v) aumento do número de plantas por área. Por outro lado, pode apresentar como fator negativo a má formação e desenvolvimento do sistema radicular, ocasionado pelo dimensionamento incorreto de recipientes
(PLATT \& OPITZ, 1973; CASTLE \& ROUSE, 1991). O apropriado dimensionamento do volume, altura e diâmetro do recipiente são variáveis conforme a espécie. A restrição do crescimento do sistema radicular, proporcionado pelo volume do recipiente, pode promover o desequilíbrio na razão entre raízes e parte aérea, alterando as respostas fisiológicas da planta (REIS et al., 1989) e repercutindo na qualidade da muda.

A qualidade do substrato constitui outro fator importante na produção de mudas. Inúmeros materiais podem ser empregados como substrato, devendo-se levar em conta a disponibilidade, custo e características físico-químicas do mesmo. Para MOURÃO FILHO et al. (1998), a correta combinação de materiais deve garantir boas características físicas, tal como boa drenagem e retenção de água.

O presente trabalho objetivou avaliar diferentes recipientes e o tipos de substrato de cultivo na produção de mudas de cancorosa e grápia.

\section{MATERIAL E MÉTODOS}

O experimento foi conduzido no Viveiro Florestal do Departamento de Ciências Florestais, vinculado ao Centro de Ciências Rurais, da Universidade Federal de Santa Maria (UFSM).

As sementes de grápia foram obtidas em Nova Prata (RS) e as de cancorosa, do Jardim Botânico da UFSM, Santa Maria (RS). A semeadura foi realizada no dia 12 de dezembro de 1997. As sementes de grápia receberam escarificação química com ácido sulfúrico concentrado, segundo método descrito por NICOLOSO et al. (1997), enquanto que as de cancorosa não sofreram nenhum tratamento pré-germinativo.

Os tratamentos consistiram de sete recipientes e três substratos.

Os recipientes usados foram os seguintes:

1) saco plástico pequeno: $10,5 \times 17,5 \mathrm{~cm}$ em diâmetro/altura, $1515 \mathrm{~cm}^{3}$ de volume;

2) saco plástico médio: 11,5 x $29,5 \mathrm{~cm}$ em diâmetro/altura, $3064 \mathrm{~cm}^{3}$ de volume;

3) saco plástico grande: 16,0 x 30,0 cm em diâmetro/altura, $6051 \mathrm{~cm}^{3}$ de volume;

4) laminado de madeira pequeno: $6,5 \times 15,0 \mathrm{~cm}$ em diâmetro/altura, $497 \mathrm{~cm}^{3}$ de volume;

5) laminado de madeira médio: $6,5 \times 25,0 \mathrm{~cm}$ em diâmetro/altura, $829 \mathrm{~cm}^{3}$ de volume;

6) laminado de madeira grande: $6,5 \times 35,0 \mathrm{~cm}$ em diâmetro/altura, $1161 \mathrm{~cm}^{3}$ de volume;

7) tubete cilíndrico: $2,5 \times 12,5 \mathrm{~cm}$ em diâmetro/altura, $50 \mathrm{~cm}^{3}$ de volume;

Os substratos usados foram os seguintes: 1) solo (horizonte A, coletado na camada de 0 a $65 \mathrm{~cm}$ de profundidade de um Podzólico vermelho 
amarelo, no município de Santa Maria, RS); 2) solo (idêntico ao anterior) + casca de arroz carbonizada; e 3) areia de granulometria média + casca de arroz carbonizada, na proporção 1:1 v/v. Foi realizada adubação nos três substratos, com adição de $2,0 \mathrm{~kg}$ do fertilizante composto NPK 5:20:20 por $\mathrm{m}^{3}$ de substrato, antes da semeadura e de modo a distribuíla uniformemente em todo volume do recipiente.

$\mathrm{O}$ delineamento experimental usado foi o de blocos ao acaso com quatro repetições, em combinação bifatorial $7 \times 3$. A parcela experimental consistiu de cinco repetições por tratamento. As mudas permaneceram por um período de 135 dias sob condição de viveiro, com cobertura de sombrite (malha $50 \%$ de extinção de luz), a $50 \mathrm{~cm}$ de altura. Quando necessário, na ausência de precipitação pluviométrica ou quando a temperatura do ar ultrapassava $25^{\circ} \mathrm{C}$, realizou-se irrigação manualmente, de tal modo que todas parcelas recebessem o mesmo volume de água.

As avaliações foram realizadas aos 135 dias da semeadura, correspondendo a 90 e 120 dias após a emergência das plântulas, respectivamente, da cancorosa e grápia. Foram avaliados o diâmetro do caule a $0,5 \mathrm{~cm}$ do substrato, altura da planta, percentagem de sobrevivência das mudas, peso da matéria seca das folhas, do caule, das raízes e total da planta, comprimento do sistema radicular (segundo TENNANT, 1975) e os aspectos visuais da parte aérea, bem como a caracterização física dos substratos (conforme metodologia descrita pela EMBRAPA, 1979).

A análise estatística dos dados foi realizada pelo teste de Duncan, em nível de 5\% de probabilidade de erro.

\section{RESULTADOS E DISCUSSÃO}

Observou-se que não houve interação significativa entre os fatores tipo e tamanho de recipiente e o tipo de substrato para nenhum dos parâmetros estudados, somente o efeito isolado desses fatores em alguns parâmetros.

\section{Efeito do tipo e tamanho de recipiente:}

Aos 90 dias após a emergência (DAE) da cancorosa, não se verificou diferença significativa de tamanho e tipo do recipiente para nenhum dos parâmetros de crescimento analisados (Tabela 1), exceto na altura, onde o saco plástico pequeno apresentou o melhor resultado e o tubete o pior. A inexistência de diferenças no desenvolvimento das plantas pode ser atribuída, parcialmente, à lenta taxa de crescimento da espécie. Quanto à sobrevivência das mudas, o laminado grande não foi adequado, enquanto o saco plástico pequeno apresentou-se superior aos laminados pequeno e médio.
Aos 120 DAE da grápia, verificou-se que, de modo geral, o saco plástico médio e grande proporcionaram valores superiores no diâmetro do caule, altura de planta, percentagem de sobrevivência das mudas, comprimento do sistema radicular, matéria seca de raízes e do caule (Tabela 2). Com base nesses resultados, deduz-se que há uma dependência parcial do crescimento das mudas de grápia em relação ao volume do recipiente. REIS et al. (1989), em estudo sobre a restrição do sistema radicular de Eucalyptus grandis, E. cloeziana e $\boldsymbol{E}$. camaldulensis, verificaram, 116 dias após a emergência das plantas, maiores taxas de crescimento da parte aérea quando submetidas aos menores níveis de restrição, permitindo às plantas usufruírem de um maior volume de substrato para o crescimento das raízes. MATTEI (1994) observou que mudas de Pinus taeda produzidas em tubetes, apresentaram menor crescimento do sistema radicular, com deformação das raízes laterais. Esse fato ocasionou problemas de adaptação das mudas, quando foram levadas a campo, com conseqüências negativas ao desenvolvimento das plantas.

HSU et al. (1996), estudando o efeito do

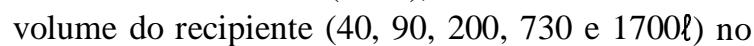
desenvolvimento de Syzygium samarangense, observaram que, após seis meses, a área transversal do tronco, a $15 \mathrm{~cm}$ acima do solo, aumentou quadraticamente com o volume do recipiente e, no final do $1^{\circ}$ e $2^{\circ}$ ano de crescimento, o número de folhas, área foliar, matéria seca das folhas, caule, ramos e das raízes foram positivamente correlacionada com o volume do recipiente. Entretanto, a razão entre a matéria seca da raiz e da parte aérea permaneceu constante entre os tratamentos durante o período experimental. Desse modo, a restrição radicular é um método efetivo para reduzir o crescimento radicular e da parte aérea de Syzygium samarangense.

Outro aspecto que, possivelmente, influenciou o desenvolvimento da grápia, além do volume do recipiente, foi a relação entre a altura e o diâmetro do mesmo. Observou-se que o peso da matéria seca de raízes e do caule foi significativamente maior no laminado grande e no saco plástico médio e grande (Tabela 2), nos quais esses recipientes, principalmente o laminado grande, apresentam maior relação entre a altura e diâmetro. Esse resultado, talvez esteja relacionado ao fato da grápia possuir sistema radicular acentuadamente pivotante e com poucas raízes secundárias na fase de plântula, assim beneficiando-se do maior comprimento do recipiente. Dados semelhantes foram obtidos por GOMES et al. (1978), em experimento realizado com Eucalyptus grandis, utilizando recipientes de plástico com diâmetros variando de 4,5 a $7,6 \mathrm{~cm}$ e 
Tabela 1 - Efeito de tipos de recipientes e substratos no crescimento de mudas de cancorosa (Maytenus ilicifolia) quanto ao diâmetro de caule, altura da planta, percentagem de sobrevivência das mudas $(\% \mathrm{~S})$, comprimento do sistema radicular (CsR), peso da matéria seca do sistema radicular (msR), do caule (msC), das folhas (msF) e total da planta (msT). UFSM, Santa Maria, 1998.

\begin{tabular}{|c|c|c|c|c|c|c|c|c|}
\hline RECIPIENTE & diâmetro (mm) & altura $(\mathrm{cm})$ & $\% \mathrm{~S}$ & $\mathrm{CsR}(\mathrm{cm})$ & msR (mg) & $\mathrm{msF}(\mathrm{mg})$ & $\mathrm{msC}(\mathrm{mg})$ & msT (mg) \\
\hline Saco plástico pequeno & $0,78 a^{*}$ & $3,3 a$ & $75,0 \mathrm{a}$ & $140,0 \mathrm{ab}$ & $40 \mathrm{a}$ & $42 a$ & $13 a$ & $95 \mathrm{a}$ \\
\hline Saco plástico médio & $0,75 a$ & $3,0 \mathrm{ab}$ & $68,3 \mathrm{ab}$ & $230,3 \mathrm{a}$ & $35 \mathrm{a}$ & $52 \mathrm{a}$ & $14 \mathrm{a}$ & $101 \mathrm{a}$ \\
\hline Saco plástico grande & $0,71 \mathrm{a}$ & $2,7 \mathrm{abc}$ & $61,7 \mathrm{ab}$ & $154,1 \mathrm{ab}$ & $54 a$ & $51 \mathrm{a}$ & $13 \mathrm{a}$ & $118 \mathrm{a}$ \\
\hline Laminado pequeno & $0,63 \mathrm{a}$ & $2,2 \mathrm{~cd}$ & $52,7 \mathrm{~b}$ & $108,5 \mathrm{~b}$ & $38 \mathrm{a}$ & $20 \mathrm{a}$ & $7 a$ & $65 \mathrm{a}$ \\
\hline Laminado médio & $0,57 \mathrm{a}$ & $2,4 \mathrm{bc}$ & $51,7 \mathrm{~b}$ & $136,2 \mathrm{ab}$ & $27 \mathrm{a}$ & $29 a$ & $14 \mathrm{a}$ & $70 \mathrm{a}$ \\
\hline Laminado grande & $0,56 a$ & $2,4 \mathrm{bc}$ & $35,0 \mathrm{c}$ & $179,9 \mathrm{ab}$ & $20 \mathrm{a}$ & $42 a$ & $7 a$ & $69 a$ \\
\hline Tubete & $0,70 \mathrm{a}$ & $1,5 \mathrm{~d}$ & $66,3 \mathrm{ab}$ & $123,7 \mathrm{ab}$ & $25 \mathrm{a}$ & $20 \mathrm{a}$ & $9 \mathrm{a}$ & $54 \mathrm{a}$ \\
\hline \multicolumn{9}{|l|}{ SUBSTRATO $^{1}$} \\
\hline $\mathrm{S}+\mathrm{C}$ & $0,75 \mathrm{a} *$ & $3,3 \mathrm{a}$ & $62,9 \mathrm{a}$ & $206,2 \mathrm{a}$ & $50 \mathrm{a}$ & $61 \mathrm{a}$ & $15 \mathrm{a}$ & $119 \mathrm{a}$ \\
\hline S & $0,67 \mathrm{ab}$ & $2,1 \mathrm{~b}$ & $51,8 \mathrm{~b}$ & $111,5 \mathrm{~b}$ & $28 \mathrm{~b}$ & $23 \mathrm{~b}$ & $10 \mathrm{~b}$ & $61 \mathrm{~b}$ \\
\hline$A+C$ & $0,57 \mathrm{~b}$ & $2,0 \mathrm{~b}$ & $61,0 \mathrm{a}$ & $138,7 \mathrm{~b}$ & $28 \mathrm{~b}$ & $20 \mathrm{~b}$ & $7 \mathrm{~b}$ & $55 \mathrm{~b}$ \\
\hline Média & 0,66 & 2,42 & 60,8 & 152,0 & 35 & 36 & 11 & 81 \\
\hline $\mathrm{CV}(\%)$ & 24,3 & 23,2 & 21,7 & 39,3 & 43,7 & 53,6 & 42,3 & 50,1 \\
\hline
\end{tabular}

*Médias não ligadas por mesma letra, na vertical, diferem pelo teste de Duncan em nível de 5\% de probabilidade de erro.

${ }^{1} \mathrm{~S}+\mathrm{C}=$ solo + casca de arroz carbonizada, $\mathrm{S}=$ solo e $\mathrm{A}+\mathrm{C}=$ areia + casca de arroz carbonizada.

altura entre 4,0 a $16,0 \mathrm{~cm}$. Esses autores concluíram que um recipiente com 5,1 x $16,0 \mathrm{~cm}$, respectivamente, em diâmetro e altura, ou seja, recipiente com pequeno diâmetro e grande altura, apresentaram as melhores características à produção de mudas.

PEZZUTTI (1998), estudando a influência de três modelos de tubete na formação de mudas de Eucalyptus globulus, aos 100 dias após a semeadura, observou que, quando foi aplicado o fertilizante composto NPK [60\% de supersimples $(0: 18: 0)$ e $40 \%$ de fertilizante de liberação lenta (14:14:14)], até a dose de $7,5 \mathrm{~kg} / \mathrm{m}^{-3}$ de substrato, os maiores crescimentos em altura, diâmetro do colo, matéria seca da parte aérea, matéria seca de raízes, volume radicular e número de folhas foram obtidos no modelo de tubete $3\left(120 \mathrm{~cm}^{3}\right.$, cônico), seguido dos modelos $2\left(56 \mathrm{~cm}^{3}\right.$, piramidal) e $1\left(50 \mathrm{~cm}^{3}\right.$, cilíndrico $)$ respectivamente. Demonstrando, desse modo, não só o efeito da disponibilidade de nutrientes em relação ao volume de substrato, mas também a influência da arquitetura do recipiente no crescimento da planta como um todo.

Pelo fato de o período experimental ter se prolongado até meados do outono, verificou-se que a grápia, por ser uma espécie caducifólia, apresentou aos 60 DAE o início da queda de folhas e aos 90 DAE mais do que $60 \%$ das plantas encontravam-se com reduzido número de folhas. Entretanto, a maior retenção foliar nas plantas cultivadas em tubetes, algo constatado na matéria seca de folhas (Tabela 2), e que se reflete no peso da matéria seca total da planta, torna-se de difícil explicação, porque as demais variáveis de crescimento analisadas desfavoreceram esse recipiente à produção de uma boa muda. Esses resultados corroboram àqueles obtidos por PEZZUTTI (1998).

\section{Efeito do tipo de substrato:}

Verificaram-se valores significativamente maiores no comprimento do sistema radicular, peso da matéria seca de raízes, do caule, das folhas e total da cancorosa quando as mudas foram cultivadas no substrato composto por solo + casca de arroz carbonizada (Tabela 1).

$\mathrm{Na}$ grápia, os substratos solo + casca de arroz carbonizada e solo, isoladamente, apresentaram resultados superiores à areia + casca de arroz carbonizada quanto ao diâmetro do caule, altura da planta, comprimento do sistema radicular e no peso da matéria seca de raízes, do caule, das folhas e total da planta. A utilização isolada de solo aumentou significativamente o comprimento do sistema radicular, matéria seca do sistema radicular e do caule, já a mistura solo + casca de arroz carbonizada proporcionou maior percentagem de sobrevivência e matéria seca de folhas (Tabela 2).

A mistura de casca de arroz carbonizada ao solo conferiu uma menor densidade ao substrato, resultando em maior porosidade (Tabela 3). O solo, de forma isolada, apresentou maior retenção de água (Tabela 3), verificado na umidade volumétrica. 
Tabela 2 - Efeito de tipos de recipientes e substratos no crescimento de mudas de grápia (Apuleia leiocarpa) quanto ao diâmetro de caule, altura da planta, percentagem de sobrevivência da mudas $(\% \mathrm{~S})$, comprimento do sistema radicular (CsR), peso da matéria seca do sistema radicular $(\mathrm{msR})$, do caule $(\mathrm{msC})$, das folhas $(\mathrm{msF})$ e total da planta (msT). UFSM, Santa Maria, 1998.

\begin{tabular}{|c|c|c|c|c|c|c|c|c|}
\hline RECIPIENTE & diâmetro (mm) & altura $(\mathrm{cm})$ & $\% \mathrm{~S}$ & $\mathrm{CsR}(\mathrm{cm})$ & $\mathrm{msR}(\mathrm{mg})$ & $\mathrm{msF}(\mathrm{mg})$ & $\mathrm{msC}(\mathrm{mg})$ & $\mathrm{msT}(\mathrm{mg})$ \\
\hline Saco plástico pequeno & $1,83 \mathrm{bc}^{*}$ & $11,6 \mathrm{bc}$ & $61,7 \mathrm{abc}$ & $179,5 \mathrm{~b}$ & $125 \mathrm{bc}$ & $82 \mathrm{cdf}$ & $133 \mathrm{bc}$ & $340 \mathrm{~cd}$ \\
\hline Saco plástico médio & $2,04 \mathrm{ab}$ & $15,8 \mathrm{a}$ & $70,0 \mathrm{a}$ & $262,3 \mathrm{a}$ & $225 \mathrm{ab}$ & $145 \mathrm{cb}$ & $222 \mathrm{a}$ & $592 \mathrm{~b}$ \\
\hline Saco plástico grande & $2,34 \mathrm{a}$ & $14,5 \mathrm{a}$ & $75,0 \mathrm{a}$ & $213,6 \mathrm{ab}$ & $224 \mathrm{ab}$ & $126 \mathrm{cb}$ & $1198 \mathrm{ab}$ & $548 \mathrm{bc}$ \\
\hline Laminado pequeno & $1,57 \mathrm{~cd}$ & $10,4 \mathrm{~cd}$ & $52,0 \mathrm{bcd}$ & $175,2 \mathrm{~b}$ & $127 \mathrm{bc}$ & $45 \mathrm{~d}$ & $87 \mathrm{c}$ & $259 \mathrm{~d}$ \\
\hline Laminado médio & $1,70 \mathrm{bcd}$ & $9,0 \mathrm{~cd}$ & $46,0 \mathrm{~cd}$ & $159,3 \mathrm{bc}$ & $174 \mathrm{bc}$ & $70 \mathrm{~cd}$ & $114 \mathrm{bc}$ & $358 \mathrm{bc}$ \\
\hline Laminado grande & $1,76 \mathrm{bcd}$ & $11,5 \mathrm{bc}$ & $40,0 \quad \mathrm{~d}$ & $186,5 \mathrm{~b}$ & $325 \mathrm{a}$ & $161 \mathrm{~b}$ & $218 \mathrm{a}$ & $704 \mathrm{~b}$ \\
\hline Tubete & $1,36 \mathrm{~d}$ & $6,6 \mathrm{~d}$ & $70,0 \mathrm{a}$ & $104,3 \mathrm{c}$ & $75 \mathrm{c}$ & $913 a$ & $54 \mathrm{c}$ & $1043 a$ \\
\hline \multicolumn{9}{|l|}{ SUBSTRATO $^{1}$} \\
\hline $\mathrm{S}+\mathrm{C}$ & $1,72 \mathrm{~b}$ & $12,6 \mathrm{a}$ & $71,7 \mathrm{a}$ & 204,4 b & $130 \mathrm{~b}$ & $531 \mathrm{a}$ & $102 \mathrm{~b}$ & $763 a$ \\
\hline $\mathrm{S}$ & $2,30 \mathrm{a}$ & $13,3 \mathrm{a}$ & $56,9 \mathrm{~b}$ & $265,0 \mathrm{a}$ & $273 a$ & $176 \mathrm{~b}$ & $246 a$ & $695 a$ \\
\hline$A+C$ & $1,19 \mathrm{c}$ & $6,6 \mathrm{~b}$ & $54,6 \mathrm{~b}$ & $82,9 \mathrm{c}$ & $48 \mathrm{c}$ & $11 \mathrm{c}$ & $38 \mathrm{c}$ & $97 \mathrm{~b}$ \\
\hline Média & 1,81 & 10,0 & 59,5 & 183,0 & 159 & 258 & 136 & 530 \\
\hline C.V. $(\%)$ & 17,5 & 24,6 & 24,6 & 38,2 & 34,3 & 25,8 & 43,3 & 39,9 \\
\hline
\end{tabular}

*Médias não ligadas por mesma letra, na vertical, diferem pelo teste de Duncan em nível de 5\% de probabilidade de erro.

${ }^{1} \mathrm{~S}+\mathrm{C}=$ solo + casca de arroz carbonizada, $\mathrm{S}=$ solo e $\mathrm{A}+\mathrm{C}=$ areia + casca de arroz carbonizada.

Segundo CASTRO \& CHEMALE (1995), a cancorosa suporta, temporariamente, solos com umidade excessiva, entretanto, observou-se menor desenvolvimento dessa espécie (Tabela 1) no substrato solo. Por outro lado, a grápia mostrou-se mais tolerante à alta umidade, adaptando-se tanto ao solo isoladamente como ao solo + casca de arroz carbonizada (Tabela 2). NICOLOSO et al. (1997) observaram maior crescimento inicial de mudas de grápia em substrato composto por areia + casca de arroz carbo-

Tabela 3 - Características físicas de diferentes substratos utilizados na produção de mudas de cancorosa (Maytenus ilicifolia) e grápia (Apuleia leiocarpa), submetidos às condições experimentais de cultivo aos 135 dias após a semeadura. UFSM, Santa Maria, 1998.

\begin{tabular}{llcl}
\hline Substrato & $\begin{array}{c}\text { Densidade } \\
\left(\mathrm{g} / \mathrm{cm}^{3}\right)\end{array}$ & $\begin{array}{c}\text { Umidade volumétrica } \\
\left(\mathrm{cm}^{3} / \mathrm{cm}^{3}\right)\end{array}$ & $\begin{array}{c}\text { Porosidade total } \\
\left(\mathrm{cm}^{3} / \mathrm{cm}^{3}\right)\end{array}$ \\
\hline $\mathrm{S}+\mathrm{C}$ & $0,873 \mathrm{~b}^{*}$ & $0,244 \mathrm{~b}$ & $0,620 \mathrm{a}$ \\
$\mathrm{S}$ & $1,360 \mathrm{a}$ & $0,321 \mathrm{a}$ & $0,549 \mathrm{~b}$ \\
$\mathrm{~A}+\mathrm{C}$ & $0,863 \mathrm{~b}$ & $0,103 \mathrm{c}$ & $0,371 \mathrm{c}$ \\
Média & 1,032 & 0,223 & 0,513 \\
C.V. $(\%)$ & 6,5 & 10,9 & 5,0 \\
\hline
\end{tabular}

*Médias não ligadas por mesma letra, na vertical, diferem pelo teste de Duncan em nível de $5 \%$ de probabilidade de erro.

${ }^{1} \mathrm{~S}+\mathrm{C}=$ solo + casca de arroz carbonizada, $\mathrm{S}=$ solo e $\mathrm{A}+\mathrm{C}=$ areia + casca de arroz carbonizada. nizada, acondicionado em caixas de madeira com $10 \mathrm{~cm}$ de altura. DUTRA \& KERSTEN (1996) verificaram que a areia + serragem, dentre nove outros substratos, proporcionaram o maior percentual de enraizamento em estacas de ameixeira (Prunus salicina Lindl.) coletadas em janeiro e março, atribuindo a essa combinação as melhores condições físicas do substrato, através do equilíbrio entre os teores de água e ar e a adequada densidade.

Além das características físicas do substrato, deve-se levar também em consideração a sua fertilidade, haja vista a grande exigência nutricional da grápia em N, P, K e S (NICOLOSO et al., 1999), o que pode estar associado ao maior crescimento da grápia nos recipientes com maior volume de substrato (Tabela 2).

GOMES et al. (1991) verificaram melhor crescimento e qualidade de mudas de Eucalyptus grandis com uso de composto orgânico em mistura com moinha de carvão. OLIVEIRA et al. (1995) observaram que a adição de matéria orgânica ao solo resultou em maior diâmetro de colo e altura de plantas de Pelthophorium dubium.

\section{CONCLUSÕES}

A combinação do recipiente saco plástico de tamanho pequeno $\left(1515 \mathrm{~cm}^{3}\right)$ e substrato solo + casca de arroz carbonizada apresenta características adequadas à produção de mudas de cancorosa (Maytenus ilicifolia). 
A combinação dos recipientes saco plástico de tamanho médio $\left(3064 \mathrm{~cm}^{3}\right)$ e grande $\left(6031 \mathrm{~cm}^{3}\right)$ e o laminado de madeira grande $\left(1161 \mathrm{~cm}^{3}\right)$ combinados com os substratos solo + casca de arroz carbonizada e solo isoladamente apresentam características adequadas à produção de mudas de grápia (Apuleia leiocarpa).

\section{REFERÊNCIAS BIBLIOGRÁFICAS}

BRASIL. Levantamento de reconhecimento dos solos do Estado do Rio Grande do Sul. Recife : Ministério da Agricultura - Departamento Nacional de Pesquisa Agropecuária Divisão de Pesquisas Pedológicas, 1973. 431p. (Boletim Técnico, 30)

CARLINI, E.A., BRAZ, S. Efeito protetor do liofilizado obtido do abafado de Maytenus ilicifolia sp. (espinheira santa) contra úlcera gástrica experimental em ratos. In: CARLINI, E.A. Estudo de ação antiúlcera gástrica de plantas brasileiras (Maytenus ilicifolia "espinheira santa" e outras). Brasília: CEME/AFIP, 1988. Cap.2. p.21-35.

CASTLE, W.S., ROUSE, R.E. Total mineral content of Florida citrus nurseries plants. Proceedings of the Florida State Horticultural Society, Florida, v.103, p.42-44, 1991.

CASTRO, L.O., CHEMALE, V.M. Plantas medicinais, condimentares e aromáticas: descrição e cultivo. Guaíba : Agropecuária, 1995. 195p

DUTRA, L.F, KERSTEN, E. Efeito do substrato e da época de coleta dos ramos no enraizamento de estacas de ameixeira (Prunus salicina Lindl.). Ciência Rural, Santa Maria, v.26, n.3, p.361-366, 1996.

EMBRAPA. Serviço nacional de levantamento e conservação do solo; manual de métodos de análise de solos. Rio de Janeiro : EMBRAPA, 1979. 650p.

GEOCZE, S., VILELA, M.P., CHAVES, B.D.R., et al Tratamento de pacientes portadores de dispepsia alta ou de úlcera péptica com preparações de espinheira-santa (Maytenus ilicifolia). In: CARLINI, E.A. Estudo de ação antiúlcera gástrica de plantas brasileiras (Maytenus ilicifolia "espinheira santa" e outras). Brasília CEME/AFIP, 1988. Cap.6. p.75-87.

GOMES, J.M., SOUZA, A.L, NETO, F.P., et al. Influência do tamanho de embalagem plástica na formação da muda de Eucalyptus grandis Hill ex Maiden. In: CONGRESSO FLORESTAL BRASILEIRO, 3, 1978, Manaus. Anais... Manaus : INPA, 1978. 456p. v.2. p.387-388.

GOMES, J.M., COUTO, L., BORGES, R.C.G., et al. Efeito de diferentes substratos na produção de mudas de Eucalyptus grandis W. Hill Ex Maiden, “win-strip”. Revista Árvore, Viçosa, v.15, p.35-42, 1991.

HSU, Y.M., TSENG, M.J., LIN, C.H. Container volume affects growth and development of wax-apple. Hort Science, Alexandria, v.11, n.7, p.1139-1142, 1996.

MATTEI, V.L. Deformações radiculares em plantas de Pinus taeda $\mathrm{L}$ produzidas em tubetes, quando comparadas com plantas originadas por semeadura direta. Ciência Florestal, Santa Maria, v.4, n.2, p.9-21, 1994.

MATTOS, N.F., GUARANHA, J. Contribuição ao estudo da grápia (Apuleia leiocarpa). Porto Alegre : Instituto de Pesquisas de Recursos Naturais Renováveis “AP”, 1983. 25p. (Boletim Técnico, 12).

MOURÃO FILHO, F.A.A., DIAS, C.T.S., SALIBE, A.A. Efeito da composição do substrato na formação de mudas de laranjeira 'pera'. Scientia Agricola, Piracicaba, v.55, n.1, p.35$42,1998$.

NICOLOSO, F.T., GARLET, A., ZANCHETTI, F., et al. Efeito de métodos de escarificação na superação da dormência de sementes e de substratos na germinação e no desenvolvimento da grápia (Apuleia leiocarpa). Ciência Rural, Santa Maria, v.27, n.3, p.419-424, 1997

NICOLOSO, F.T., ZANCHETTI, F., GARLET, A, et al. Exigências nutricionais da grápia (Apuleia leiocarpa Vog. Macbride) em solo Podzólico vermelho amarelo. Ciência Rural, Santa Maria, v.29, n.2, p.225-231, 1999.

OLIVEIRA, O.S., WATZLAWICK, L.F., KURTZ, F.C., et al. Efeito do substrato no desenvolvimento de mudas de Pelthophorum dubium a nível de viveiro. In: CONGRESSO BRASILEIRO DE CIÊNCIA DO DO SOLO, 25, 1995, Viçosa, MG. Resumos... Viçosa : SBCS-UFV, 1995. 1158p. v.2. p.779-781.

PEZZUTTI, R.V. Formação de mudas de Eucaplyptus globulus em função da fertilização NPK, do modelo de tubete e da poda química de raiz. Santa Maria, RS, 1998. 73p. Dissertação (Mestrado em Engenharia Florestal) - Curso de Pósgraduação em Engenharia Florestal, Universidade Federal de Santa Maria, 1998.

PLATT, R.G., OPITZ, K.W. The propagation of citrus. In: PLATT, R.G., OPITZ, K.W., JORDAM, L.S., et al. The citrus indrustry. Berkeley : Universty of California, Division of Agricultural Sciences, 1973. Cap.1. p.1-47.

REIS, G.G., REIS, M.G.F., MAESTRI, M., et al. Crescimento de Eucalyptus camaldulensis, E. grandis e E. cloensiana sob diferentes níveis de restrição radicular. Revista Árvore, Viçosa, v.13, n.1, p.1-18, 1989.

TENNANT, D.A. A Test of a modified line intersect method of estimating root lenght. The Journal of Ecology, [s.1.],v.63, n.3, p.995-1001, 1975.

Ciência Rural, v. 30, n. 6, 2000. 